\title{
A case of autism spectrum disorder arising from a de novo missense mutation in POGZ
}

\author{
Ryoko Fukai $^{1,2}$, Yoko Hiraki ${ }^{3}$, Hiroko Yofune ${ }^{4}$, Yoshinori Tsurusaki ${ }^{1}$, Mitsuko Nakashima ${ }^{1}$, Hirotomo Saitsu ${ }^{1}$, \\ Fumiaki Tanaka $^{2}$, Noriko Miyake ${ }^{1}$ and Naomichi Matsumoto ${ }^{1}$ \\ Autism spectrum disorder (ASD) is a clinically heterogeneous psychiatric disorder with various genetic backgrounds. Here, we \\ report a novel mutation in the pogo transposable element-derived protein with zinc finger domain gene (POGZ) identified by trio- \\ based whole exome sequencing. To date, a total of seven de novo POGZ mutations in ASD have been reported. POGZ contains a \\ total of five functional domains, and this study reports the first de novo missense mutation in the centromere protein B-like \\ DNA-binding domain. POGZ is highly expressed in the human fetal brain and is involved in mitosis and the regulation of \\ neuronal proliferation. Therefore its loss-of-function or pathogenic missense mutations are likely to be causative of ASD. \\ Journal of Human Genetics (2015) 60, 277-279; doi:10.1038/jhg.2015.13; published online 19 February 2015
}

\section{INTRODUCTION}

Autism spectrum disorder (ASD) is characterized by deficits in social communication and interactions, stereotyped or repetitive behaviors, and restricted interests. ${ }^{1}$ The prevalence of ASD is estimated to be 62 in 10000 and the diagnosed population has increased during the last decade. $^{2}$ The clinical heterogeneity of ASD may be reflected by its heterogeneous genetic complexity. ${ }^{3}$ More than 100 autism-related genes, copy number variations and 158 linked regions have been reported. ${ }^{1,4}$ To date, at least six large-scale trio- or quads-based ASD whole exome sequencing (WES) studies have discovered de novo mutations in various genes that are causative of ASD. ${ }^{3,5-9}$ Mutant genes in ASD can be also associated with intellectual disability (ID) and epilepsy. ${ }^{3,5,10}$ Moreover, de novo loss-of-function (LoF) single nucleotide variants and insertions/deletions are observed at higher frequencies in ASD individuals than in controls. ${ }^{5-9,11}$ Genes showing LoF mutations in schizophrenia are also found as de novo mutations in ID and ASD, including the sodium channel, voltage-gated, type II, alpha subunit gene. ${ }^{12}$ Recently, the pogo transposable element-derived protein with zinc finger domain gene $(P O G Z)$ has become a plausible candidate gene for ASD as de novo mutations were identified in at least seven independent ASD patients by WES studies. ${ }^{710-12}$ Here, we report a Japanese individual with ASD possessing a novel de novo missense mutation in POGZ detected by trio-based WES.

\section{MATERIALS AND METHODS}

Case report

The patient was born at 39 weeks of gestation to healthy, non-consanguineous Japanese parents as the first child after a normal pregnancy. His birth weight was $3715 \mathrm{~g}(+1.79$ s.d. $)$, height $48.6 \mathrm{~cm}(-0.24$ s.d. $)$ and occipital-frontal circumference $34.0 \mathrm{~cm}$ ( +0.44 s.d.). On examination at 5 years of age, his height was $106.2 \mathrm{~cm}(-0.21 \mathrm{s.d}$.), weight $17.9 \mathrm{~kg}$ ( +0.26 s.d.), and occipitalfrontal circumference $46.5 \mathrm{~cm}$ ( -2.69 s.d.). He spoke no meaningful words, had a severe ID, limited social interactions, communication difficulties and repetitive behavior, and demonstrated an interest in water, and shiny or spinning objects. He was diagnosed with ASD showing level-3 social communications and level- 3 restricted interests and repetitive behaviors both requiring very substantial support, and global developmental delay according to the Fifth Edition of the Diagnostic and Statistical Manual of Mental Disorders (DSM-5). Based on the Enjoji developmental assessment, ${ }^{13}$ his developmental quotient was 16 (at age 5, motor development was judged to be at the 15-month-old level, daily living activities at 11.5 months and communication at 6.5 months). Dysmorphic facial features were noted including strabismus, a broad, low nasal bridge, anteverted nares, a prominent cupid bow and upturned corner of the mouth, and edematous hands (Figure 1). G-banded chromosomal analysis showed 46,XY,inv(9)(p12q13), which was derived from his mother with the normal phenotype. Brain magnetic resonance imaging at the age of 18 months and electroencephalography at 3 years were both normal.

\section{Whole exome sequencing}

Genomic DNA was extracted from the peripheral blood of the patient and his parents. Approximately $3 \mu \mathrm{g}$ was sheared and coding regions captured using a SureSelect Human All Exon V4 (51 Mb) library (Agilent Technologies, Santa Clara, CA, USA) according to the manufacturer's instructions. Captured DNA was sequenced on the HiSeq2000 (Illumina, Inc., San Diego, CA, USA) with $101 \mathrm{bp}$ paired-end reads. Image analysis and base calling were performed by sequence control software real-time analysis and CASAVA software v1.8 (Illumina, Inc.). The quality controlled (Path Filter) reads were mapped to the human reference genome (UCSC hg19, NCBI build 37), using Novoalign 2.08.02 (http://www.novocraft.com/). After the removal of PCR duplication by

${ }^{1}$ Department of Human Genetics, Yokohama City University Graduate School of Medicine, Yokohama, Japan; ${ }^{2}$ Department of Neurology and Stroke Medicine, Yokohama City University Graduate School of Medicine, Yokohama, Japan; ${ }^{3}$ Hiroshima Municipal Center for Child Health and Development, Hiroshima, Japan and ${ }^{4}$ Hiroshima City Hokubu Center for Children's Treatment and Guidance, Hiroshima, Japan

Correspondence: Dr N Miyake or Dr N Matsumoto, Department of Human Genetics, Yokohama City University Graduate School of Medicine, Fukuura 3-9, Kanazawa-ku, Yokohama 2360004, Japan.

E-mail: nmiyake@yokohama-cu.ac.jp or naomat@yokohama-cu.ac.jp

Received 2 December 2014; revised 13 January 2015; accepted 21 January 2015; published online 19 February 2015 
Picard 1.55 (http://broadinstitute.github.io/picard/), single nucleotide variants and short insertions and deletions (Indels) were called using Genome Analysis Toolkit (GATK) 1.6-5 (http://www.broadinstitute.org/gatk/). Called single nucleotide variants and Indels were annotated using ANNOVAR (http:// www.openbioinformatics.org/annovar/). Through this flow, common variants registered in dbSNP137 (minor allele frequency $\geqslant 0.01$ ) were removed. Of all variants within exons or $\pm 30 \mathrm{bp}$ from exon-intron boundaries, those registered in dbSNP137, the National Heart Lung and Blood Institute Exome Sequencing Project Exome Variant Server (NHLBI-ESP 6500, http://evs.gs.washington.edu/ $\mathrm{EVS} /$ ) and our in-house (exome data from 575 Japanese individuals) databases were removed. The variants were confirmed by Sanger sequencing using an ABI PRISM 3500xl autosequencer (Life Technologies, Carlsbad, CA, USA).

\section{RESULTS AND DISCUSSION}

To explore the genetic basis of ASD, we performed trio-based WES involving the patient and both parents. The mean depth of the RefSeq coding sequence was 125.11-164.12 reads, with $91.3-93.1 \%$ being covered by $\geqslant 20$ reads. One de novo missense mutation was detected and predicted as pathogenic by SIFT, ${ }^{14}$ PolyPhen $2,{ }^{15}$ and MutationTaster $^{16}$ (Table 1): POGZ NM_015100.3: c.3118G $>$ A (p.Glu1040Lys). Sanger sequencing confirmed the mutation to be de novo.

During the last five years, de novo mutations in various genes have been implicated as causative of ASD. ${ }^{17}$ Interpretation of the pathogenicity of these mutations in neuropsychiatric diseases was performed by protein-protein interaction analysis and expression studies, which indicated that $P O G Z$ is a transcriptional regulator gene in neuronal networks. ${ }^{9,18}$ It encodes a heterochromatin protein $1 \alpha$-binding protein containing a cluster of multiple $\mathrm{C} 2 \mathrm{H} 2$-type zinc fingers that may regulate gene expression, ${ }^{19}$ a centromere protein (CENP) B-like
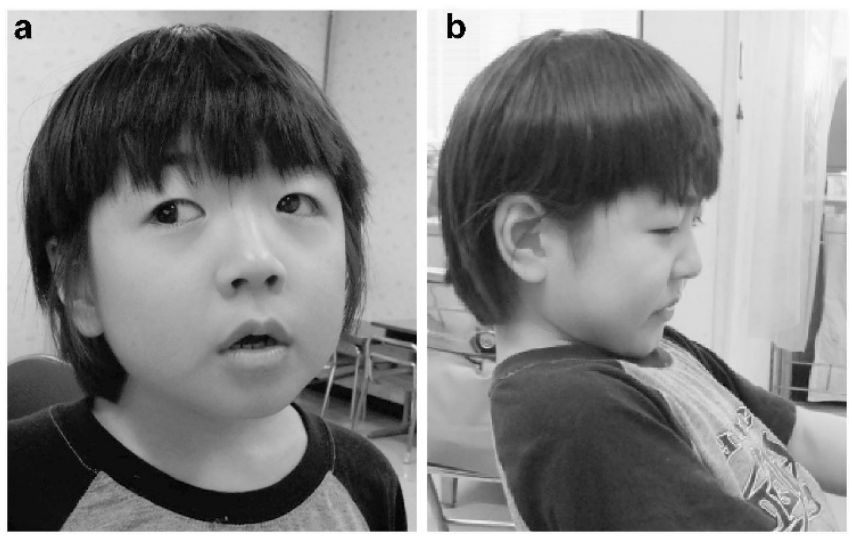

Figure 1 Craniofacial features of the patient at 5 years of age ((a) frontal view and (b) lateral view) demonstrating broad and low nasal bridge, anteverted nares, prominent cupid bow and upturned corner of the mouth. A full color version of this figure is available at the Journal of Human Genetics journal online.
DNA-binding domain, and a DDE domain (Figure 2a). ${ }^{20}$ POGZ is involved in mitosis and neuronal proliferation, ${ }^{20}$ so mutations in this gene may cause dysregulation of the neuronal proliferation associated with ASD.

To date, seven de novo POGZ mutations have been reported: three frameshifts, two stop-gain and two missense mutations have been detected on the screening for ASD, ID or schizophrenia, though clinical information was limited. ${ }^{7,10-12}$ One of the missense mutations (p.Tyr597Cys) within a zinc finger domain showed a highly pathogenic predicted score, while the other was predicted to be a benign amino acid change and occurred outside the functional domains (Table 1). ${ }^{11}$ Here, we report the first de novo missense mutation in the CENP-B-like domain, which is predicted to be highly pathogenic (Figure 2).

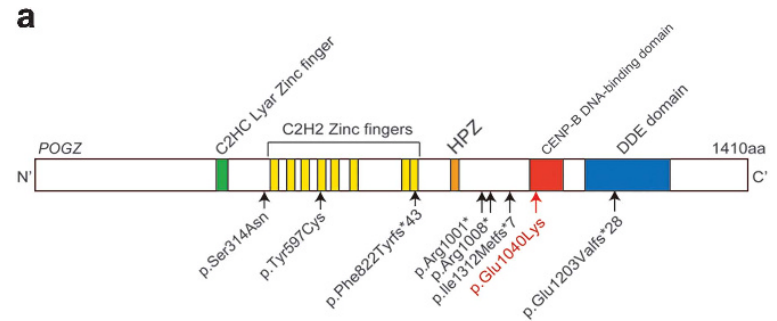

b

\begin{tabular}{ll|l|l} 
Human & $\mathrm{R}$ & $\mathrm{E}$ & $\mathrm{Q}$ \\
Rhesus & $\mathrm{R}$ & $\mathrm{E}$ & $\mathrm{Q}$ \\
Mouse & $\mathrm{R}$ & $\mathrm{E}$ & $\mathrm{Q}$ \\
Dog & $\mathrm{R}$ & $\mathrm{E}$ & $\mathrm{Q}$ \\
Elephant & $\mathrm{R}$ & $\mathrm{E}$ & $\mathrm{Q}$ \\
Opossum & $\mathrm{R}$ & $\mathrm{E}$ & $\mathrm{Q}$ \\
Chicken & $\mathrm{R}$ & $\mathrm{E}$ & $\mathrm{Q}$ \\
X-tropicalis & $\mathrm{R}$ & $\mathrm{E}$ & $\mathrm{Q}$ \\
Zebrafish & $\mathrm{R}$ & $\mathrm{E}$ & $\mathrm{Q}$
\end{tabular}

c

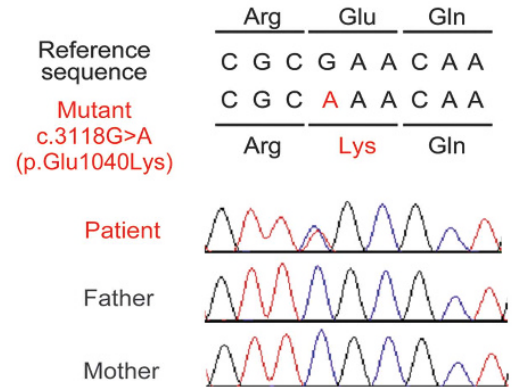

Figure 2 Schematic presentation of the POGZ protein and location of its mutations. (a) Schematic of POGZ and its five functional domains. The novel mutation identified in our patient (red) and the seven known mutations are shown below the protein. (b) Electropherograms of the patient and his parents showing the presence of the de novo mutation. (c) Interspecies evolutionary conservation of the original amino acid residue altered by the de novo mutation. POGZ, pogo transposable element-derived protein with zinc finger domain gene.

Table 1 Functional predictions of three de novo missense mutations in POGZ

\begin{tabular}{|c|c|c|c|c|c|c|c|}
\hline Gene & Accession no. & Mutation & Amino-acid change & SIFT & PolyPhen2 & MutationTaster & Patient \\
\hline POGZ & NM_015100.3 & c. $3118 \mathrm{G}>\mathrm{A}$ & Glu1040Lys & 0.01 (damaging) & 0.998 (probably damaging) & 0.99999 (disease causing) & Present case \\
\hline POGZ & NM_015100.3 & c. $1790 A>G$ & Tyr597Cys & 0 (damaging) & 0.999 (probably damaging) & 0.997 (disease causing) & lossifov et al., ${ }^{11}$ \\
\hline POGZ & NM_015100.3 & c. $941 \mathrm{G}>\mathrm{A}$ & Ser314Asn & 0.211 (tolerated) & 0.09 (benign) & 0.853 (polymorphism) & lossifov et al., ${ }^{11}$ \\
\hline
\end{tabular}

Abbreviation: POGZ, pogo transposable element-derived protein with zinc finger domain gene.

P.Glu1040Lys was found in the present patient and the other two were reported previously. 
In conclusion, we identified a novel de novo POGZ mutation that appears to be causative of ASD. Future studies should accumulate more ASD patients harboring POGZ mutations to delineate phenotypic features by $P O G Z$ abnormality.

\section{CONFLICT OF INTEREST}

The authors declare no conflict of interest.

\section{ACKNOWLEDGEMENTS}

We thank the patient's family for participating in this work. This work was supported by research grants from the Ministry of Health, Labour and Welfare of Japan (HS, N Matsumoto, N Miyake), the fund for Creation of Innovation Centers for Advanced Interdisciplinary Research Areas Program in the Project for Developing Innovation Systems from the Japan Science and Technology Agency (N Matsumoto), the Strategic Research Program for Brain Sciences (N Matsumoto) and a Grant-in-Aid for Scientific Research on Innovative areas-(Transcription cycle)—from the Ministry of Education, Culture, Sports, Science and Technology of Japan (N Matsumoto), Grants-inAid for Scientific Research (A) and (B) from the Japan Society for the Promotion of Science (HS, N Miyake, N Matsumoto), the Takeda Science Foundation (N Matsumoto, N Miyake), the Yokohama Foundation for Advancement of Medical Science (N Miyake) and the Hayashi Memorial Foundation for Female Natural Scientists (N Miyake).

1 Betancur, C. Etiological heterogeneity in autism spectrum disorders: more than 100 genetic and genomic disorders and still counting. Brain Res. 1380, 42-77 (2011).

2 Elsabbagh, M., Divan, G., Koh, Y. J., Kim, Y. S., Kauchali, S., Marcin, C. et al. Global prevalence of autism and other pervasive developmental disorders. Autism Res. 5, 160-179 (2012).

3 Persico, A. M. \& Napolioni, V. Autism genetics. Behav. Brain Res. 251, 95-112 (2013).

4 Xu, L. M., Li, J. R., Huang, Y., Zhao, M., Tang, X. \& Wei, L. AutismKB: an evidencebased knowledgebase of autism genetics. Nucleic Acids Res. 40, D1016-D1022 (2012).
5 Buxbaum, J. D., Daly, M. J., Devlin, B., Lehner, T., Roeder, K. \& State, M. W. The autism sequencing consortium: large-scale, high-throughput sequencing in autism spectrum disorders. Neuron 76, 1052-1056 (2012).

6 O'Roak, B. J., Vives, L., Girirajan, S., Karakoc, E., Krumm, N., Coe, B. P. et al. Sporadic autism exomes reveal a highly interconnected protein network of de novo mutations. Nature 485, 246-250 (2012).

7 Neale, B. M., Kou, Y., Liu, L., Ma'ayan, A., Samocha, K. E., Sabo, A. et al. Patterns and rates of exonic de novo mutations in autism spectrum disorders. Nature 485, 242-245 (2012)

8 Sanders, S. J., Murtha, M. T., Gupta, A. R., Murdoch, J. D. Raubeson, M. J. Willsey, A. J. et al. De novo mutations revealed by whole-exome sequencing are strongly associated with autism. Nature 485, 237-241 (2012).

9 De Rubeis, S., He, X., Goldberg, A. P., Poultney, C. S., Samocha, K., Cicek, A. E. et al. Synaptic, transcriptional and chromatin genes disrupted in autism. Nature $\mathbf{5 1 5}$, 209-215 (2014).

10 Gilissen, C., Hehir-Kwa, J. Y., Thung, D. T., van de Vorst, M., van Bon, B. W. M., Willemsen, M. H. et al. Genome sequencing identifies major causes of severe intellectual disability. Nature 511, 344-347 (2014)

11 lossifov, I., O'Roak, B. J., Sanders, S. J., Ronemus, M., Krumm, N., Levy, D. et al. The contribution of de novo coding mutations to autism spectrum disorder. Nature 515, 216-221 (2014).

12 Fromer, M., Pocklington, A. J., Kavanagh, D. H., Williams, H. J., Dwyer, S., Gormley, P. et al. De novo mutations in schizophrenia implicate synaptic networks. Nature 506, 179-184 (2014).

13 Enjoji, M. \& Yanai, N. Analytic test for development in infancy and childhood. Pediatr. Int. 4, 2-6 (1961).

$14 \mathrm{Ng}$, P. C. \& Henikoff, S. SIFT: Predicting amino acid changes that affect protein function. Nucleic Acids Res. 31, 3812-3814 (2003).

15 Adzhubei, I. A., Schmidt, S., Peshkin, L., Ramensky, V. E., Gerasimova, A., Bork, P. et al. A method and server for predicting damaging missense mutations. Nat. Methods 7, 248-249 (2010).

16 Schwarz, J. M., Rodelsperger, C., Schuelke, M. \& Seelow, D. MutationTaster evaluates disease-causing potential of sequence alterations. Nat. Methods 7 , 575-576 (2010).

17 Ronemus, M., Iossifov, I., Levy, D. \& Wigler, M. The role of de novo mutations in the genetics of autism spectrum disorders. Nat. Rev. Genet. 15, 133-141 (2014).

18 Liu, L., Lei, J., Sanders, S. J., Willsey, A. J., Kou, Y., Cicek, A. E. et al. DAWN: a framework to identify autism genes and subnetworks using gene expression and genetics. Mol. Autism 5, 22 (2014).

19 luchi, S. Three classes of $\mathrm{C} 2 \mathrm{H} 2$ zinc finger proteins. Cell. Mol. Life Sci. 58, 625-635 (2001)

20 Nozawa, R. S., Nagao, K., Masuda, H. T., Iwasaki, O., Hirota, T., Nozaki, N. et al. Human POGZ modulates dissociation of HP1alpha from mitotic chromosome arms through Aurora B activation. Nat. Cell Biol. 12, 719-727 (2010). 